\title{
8
}
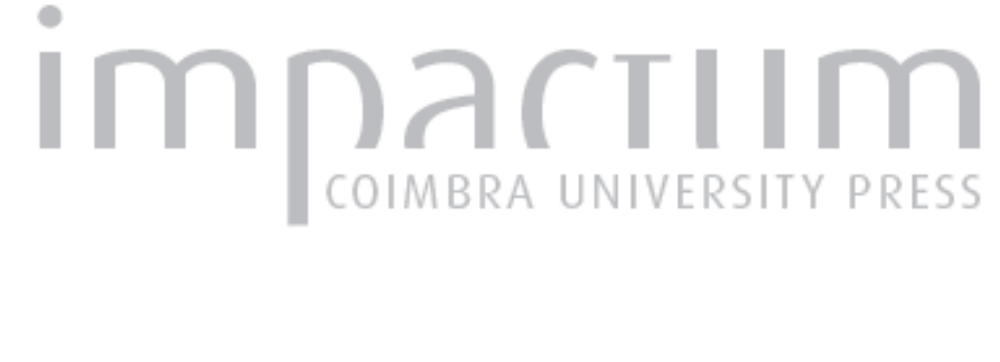

\section{Hábitos alimentares de universitários da Amazônia Brasileira}

\begin{abstract}
Guimarães, Raissa Cecília Rosalino; Valente, Glaucyellen Ferreira; Autor(es): $\quad$ Silva, Jucilane do Socorro Feitosa da; Palheta, Renata Camily Siqueira; Castilho, Silviane Ribeiro; Silva, Hilton Pereira da
\end{abstract}

Publicado por: Imprensa da Universidade de Coimbra

URL persistente:

URI:http://hdl.handle.net/10316.2/39078

DOI:

DOI:http://dx.doi.org/10.14195/2182-7982_31_5

Accessed : $\quad$ 26-Apr-2023 15:05:20

A navegação consulta e descarregamento dos títulos inseridos nas Bibliotecas Digitais UC Digitalis, UC Pombalina e UC Impactum, pressupõem a aceitação plena e sem reservas dos Termos e Condições de Uso destas Bibliotecas Digitais, disponíveis em https://digitalis.uc.pt/pt-pt/termos.

Conforme exposto nos referidos Termos e Condições de Uso, o descarregamento de títulos de acesso restrito requer uma licença válida de autorização devendo o utilizador aceder ao(s) documento(s) a partir de um endereço de IP da instituição detentora da supramencionada licença.

Ao utilizador é apenas permitido o descarregamento para uso pessoal, pelo que o emprego do(s) título(s) descarregado(s) para outro fim, designadamente comercial, carece de autorização do respetivo autor ou editor da obra.

Na medida em que todas as obras da UC Digitalis se encontram protegidas pelo Código do Direito de Autor e Direitos Conexos e demais legislação aplicável, toda a cópia, parcial ou total, deste documento, nos casos em que é legalmente admitida, deverá conter ou fazer-se acompanhar por este aviso.

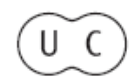




$$
\bar{A}
$$




\section{Hábitos alimentares de universitários da Amazônia Brasileira}

\section{Raissa Cecília Rosalino Guimarães ${ }^{1}$, Glaucyellen Ferreira Valente ${ }^{2}$, Jucilane do Socorro Feitosa da Silva ${ }^{2}$, Renata Camily Siqueira Palheta ${ }^{2}$, Silviane Ribeiro Castilho $^{2}$, Hilton Pereira da Silva ${ }^{3}$}

raissa_guimaraes28@hotmail.com

DOI: http://dx.doi.org/10.14195/2182-7982_31_5

Resumo Objetivo: Descrever e analisar os hábitos alimentares de uma amostra de estudantes de ambos os sexos e diferentes faixas etárias da Universidade Federal do Pará (UFPA), campus de Belém, Amazônia, Brasil. Introdução: Os hábitos alimentares são construídos durante a infância e adolescência e, com o ingresso na universidade, fatores como ambiente social e conveniência passam a condicionar fortemente estes hábitos. Em geral, estudos no Brasil demonstram inadequação do consumo

\begin{abstract}
Objective: To describe and analyze the eating habits of a sample of students of both sexes and different age groups of the Universidade Federal do Pará (UFPA), Belém, Amazonia, Brazil. Introduction: Eating habits are developed during infancy and adolescence, and with entrance into the university factors such as social environment and convenience begin to strongly condition eating behavior. In general, studies of other regions of Brazil show a quite inadequate consumption of micro- and macronutrients among
\end{abstract}

\footnotetext{
1 Universidade Federal do Pará, Mestrado em Saúde, Ambiente e Sociedade na Amazônia, Belém, PA, Brasil. ${ }^{2}$ Nutricionista.

${ }^{3}$ Universidade Federal do Pará, Programa de Pós-Graduação em Antropologia e Mestrado em Saúde, Ambiente e Sociedade na Amazônia, Laboratório de Estudos Bioantropológicos em Saúde e Meio Ambiente, Belém, PA, Brasil.
} 
alimentar de macro e micronutrientes entre universitários, podendo acarretar prejuízos futuros na saúde destes indivíduos. No entanto, não há estudos sobre os hábitos alimentares de estudantes universitários na Amazônia. Métodos: Trata-se de um estudo de delineamento transversal, qualitativo, em que 22 estudantes de graduação da UFPA, com idades entre 18 e 27 anos, selecionados aleatoriamente no campus, responderam a um questionário semiestruturado sobre frequência e comportamento alimentar. A amostragem foi encerrada usando a técnica de saturação e a interpretação das informações seguiu a técnica de análise de conteúdo. Resultados: A maioria dos universitários realiza suas refeições em número e intervalo de tempo adequado, correspondendo a parcelas de 54,5\% e 59,0\% da amostra, respectivamente. No entanto, destaca-se também o elevado consumo de alimentos com alto teor calórico; 81,8\% dos jovens entrevistados ingeriam diariamente alimentos pertencentes ao grupo das gorduras, óleos e açúcares. Os estudantes também apresentaram baixo consumo de nutrientes essenciais ao organismo, representados pelos grupos das hortaliças/ verduras e das frutas. Apenas 50,0\% e 31,1\% dos jovens, respectivamente, incorporavam estes alimentos na sua dieta habitual. Outro achado importante é que a maioria dos estudantes (59,1\%) não se preocupa com o valor nutricional dos alimentos na hora de fazer as refeições. Conclusão: Um melhor conhecimento sobre o comportamento alimentar dos universitários é necessário para o planejamento de políticas públicas de prevenção das doenças crônicas. O trabalho integrado de nutricionistas e

university students, which can be detrimental to their health in the future. However, there are no studies of eating habits of Amazonian college students. Methods: The study design was transversal and qualitative. Twenty two randomly selected graduate students from UFPA, between 18 and 27 years old answered a semi-structured questionnaire designed to investigate eating habits and frequency. The sample was closed by the technique of saturation and the answers were analyzed using the technique of content analysis. Results: The majority of college students interviewed have meals in adequate numbers and time periods daily, corresponding to $54.5 \%$ and $59.0 \%$ of the sample respectively. However, most eat large amounts of foods rich in calories; $81.8 \%$ ingest foods from the fats, oils and sugars group. Students also show low intake of essential nutrients supplied by the vegetables and fruits groups. Only 50.0\% and 31.1\% of students incorporate these foods, respectively, into their daily diet. It was noticeable among the majority of students (59.1\%) a lack of concern regarding the nutritional value of the food they eat daily. Conclusion: Better knowledge of the eating behaviors of college students is necessary to improve the public-policy planning for the prevention of chronic diseases. In addition, the integrated work of nutritionists and bioanthropologists in the area of collective/public health may be fundamental to the comprehension and promotion of change in eating patterns among young students. 
bioantropólogos na área de saúde coletiva pode ser fundamental para a compreensão e a promoção de mudanças nos padrões alimentares entre os jovens universitários.

Palavras-Chave: Universitários; hábitos alimentares; saúde pública; Amazônia; pesquisa qualitativa.

\section{Introdução}

O conhecimento sobre as práticas alimentares é formado desde os primeiros anos de vida do indivíduo uma vez que o controle do consumo alimentar é complexo devido a interação entre fatores culturais, ambientais, sociais e religiosos que produzem diferentes respostas em relação ao ato de comer, sendo influenciado por estes fatores ao longo de toda a vida adulta (Oliveira et al., 2012).

Após a primeira infância, as novas preferências alimentares são condicionadas, em geral, devido ao crescimento autônomo e a oportunidade de se alimentar, também, longe da família. Assim, ao longo da juventude o comportamento alimentar vai se delineando pela escolha particular dos alimentos, sendo influenciado por diferentes componentes: o cognitivo (correspondente àquilo que o indivíduo sabe sobre alimentos e nutrição), o afetivo (correspondente àquilo que sentimos em relação aos alimentos e as práticas alimentares) e o situacional
Keywords: University students; eating habits; public health; Amazonia; qualitative research.

(fatores que podem facilitar ou dificultar o acesso e a ingestão de alimentos) (Cambraia et al., 2012).

Já é amplamente reconhecido que os padrões de ingestão alimentar durante a infância e adolescência podem predizer a ocorrência de obesidade e doenças cardiovasculares na idade adulta, bem como determinar o risco de alguns tipos de câncer relacionados à dieta e as mudanças nos padrões de alimentação em muitos países (Hu, 2008). Também já é reconhecido que o aumento do consumo de fast-food, refeições pré-preparadas e refrigerantes tem tornado as escolhas alimentares saudáveis mais difíceis para todas as faixas etárias (Neutzling et al., 2010).

Estudantes universitários estão geralmente em um período da vida em que hábitos e atitudes estão em transição entre os do tempo da infância/adolescência, mais voltados para a vida doméstica e escolar, e os da idade adulta, mais voltados para a carreira e a constituição de suas próprias relações sociais/familiares. 
Neste período, as preferências alimentares são fortemente influenciadas pelas circunstâncias ecológicas e pelo ambiente social com os quais os indivíduos são obrigados a conviver durante grande parte do seu dia na universidade (Azevedo et al., 2008).

Os principais determinantes das escolhas alimentares destes jovens são o tempo disponível, a conveniência, o custo, o sabor e o ambiente social e físico onde se realizam as refeições, fatores que podem propiciar a substituição de refeições completas por lanches, considerados mais práticos e rápidos, mas que contêm alto valor calórico; soma-se a isso a questão da disponibilidade ou não de alimentos saudáveis no campus e o estabelecimento de novos comportamentos e relações sociais pré-profissionais (Martins, 2009).

Estudos fora da região amazônica demonstram que a ingestão alimentar dos estudantes universitários normalmente não satisfaz as recomendações para a maioria dos grupos de alimentos e para os principais micronutrientes, o que constitui motivo de preocupação para a saúde pública, uma vez que os comportamentos, crenças e conhecimentos sobre a alimentação desenvolvidos e exibidos durante este período de vida irão repercutir na idade adulta e influenciar o estado de saúde futuro (Martins, 2009).

Este artigo tem como objetivo descrever e analisar os hábitos alimentares de uma amostra de estudantes de ambos os sexos e diferentes faixas etárias da Universidade Federal do Pará (UFPA), campus de Belém, Amazônia, na perspectiva de contribuir para a ampliação dos conhecimentos sobre a alimentação de jovens adultos no Brasil.

\section{Métodos}

Foi realizado um estudo transversal, exploratório, de natureza qualitativa, com amostragem aleatória estratificada simples, envolvendo 22 estudantes de graduação de ambos os sexos, na faixa etária de 18 a 27 anos, matriculados regularmente em diferentes cursos ofertados pela Universidade Federal do Pará (UFPA), campus de Belém, Amazônia, Brasil, durante o mês de outubro de 2011. A UFPA é a principal instituição de ensino superior e pesquisa da região Amazônica, sendo o campus de Belém, capital do estado do Pará, o maior entre os nove que compõem a instituição.

$O$ instrumento utilizado na pesquisa foi um questionário semiestruturado desenvolvido especialmente para este estudo, contendo perguntas sobre os principais alimentos consumidos por eles no seu cotidiano, a frequência (diária, semanal, mensal) de ingestão desses alimentos, como dividem suas refeições ao longo do dia e o intervalo com que fa- 
zem tais refeições. Para a análise, as questões foram organizadas por grupos alimentares, divididos da seguinte maneira: grupo 1 (pães, arroz, cereais e massas); grupo 2 (hortaliças e vegetais); grupo 3 (frutas); grupo 4 (leite, iogurtes, queijos e derivados); grupo 5 (carnes, aves, peixes); grupo 6 (feijão e leguminosas) e grupo 7 (gorduras, óleos e açúcares).

Os entrevistados também responderam a questões sobre seus comportamentos alimentares, como a preocupação com o valor nutricional na hora da aquisição de determinado alimento; costume de consumir líquidos durante as refeições e quais; e quantidade de ingestão diária de água.

O consumo de porções diárias dos grupos dos alimentos foi comparado às orientações sugeridas pela Pirâmide Adaptada para a População Brasileira (Philippi, 2008) e a avaliação dos hábitos alimentares foi baseada nas recomendações do Guia Alimentar para a População Brasileira (Brasil, 2008).

Dentre os estudantes, recrutados aleatoriamente nos diversos espaços do campus da universidade, 12 (54,5\%) eram do sexo masculino e $10(45,4 \%)$ do sexo feminino. A amostragem foi encerrada pela técnica de saturação, procedimento de análise qualitativa na qual o pesquisador busca identificar em qual ponto (neste caso, número de participantes) as respostas obtidas não apresentam mais informações novas, isto é, estão "saturadas" (Mason, 2010), e a interpretação das informações seguiu a técnica de análise de conteúdo, que utiliza análises sistemáticas para compreender o pensamento do sujeito a partir do conteúdo de sua informação registrada na entrevista (Bardin, 1977; Krippendorff, 2003; Guerra, 2008).

Os universitários foram abordados anteriormente sobre o propósito da pesquisa e, após os esclarecimentos metodológicos e éticos, assinaram um termo de consentimento concordando em participar da entrevista. Os inquéritos foram anônimos, para conferir confidencialidade à informação coletada e a pesquisa seguiu os preceitos éticos da Resolução 466/12 do Conselho Nacional de Saúde do Brasil.

\section{Resultados}

Os universitários entrevistados apresentaram média de idade de 21,70 anos. Ao serem questionados quanto ao local onde costumam realizar as refeições, $27,2 \%$ responderam que era em casa, $22,7 \%$ no restaurante universitário (RU) da UFPA e 50,0\% comem em ambos os locais.

O número de refeições realizadas por dia pelos estudantes varia de 2 a 8 . A maioria faz de 3 a 4 refeições (54,5\%); seguidos dos que fazem de 5 a 6 refei- 
ções $(27,7 \%)$, de 13,6\% que fazem de 7 a 8 refeições diárias, e de 9,0\% que realizam apenas 2 refeições por dia. Estas refeições são distribuídas em desjejum, colação, almoço, lanche, jantar e ceia, observando-se o predomínio da realização do desjejum (90,9\%), almoço (100\%) e jantar (90,9\%).

O intervalo de tempo entre as refeições varia de 2 a 9 horas. Há 9,0\% que ficam aproximadamente 2 horas sem se alimentar; $59,0 \%$ de 3 a 4 horas; seguidos de $27,2 \%$ que ficam de 5 a 6 horas e 4,5\% que ficam até 9 horas seguidas sem se alimentar.

Ao serem questionados se há alguma preocupação com o valor nutricional dos alimentos na hora da compra, 40,9\% responderam que sim e 59,1\% que não. Durante as refeições, 77,2\% relataram consumir líquidos e apenas 27,2\% não têm o hábito de ingerir líquidos.

A ingestão alimentar é descrita a seguir de acordo com a frequência de consumo dos alimentos por grupos, estratificado por seu consumo diário, semanal, mensal ou nunca.

O consumo de alimentos do grupo 1 (pães, cereais e massas) apresentou frequência diária por 90,9\% dos estudantes, variando de 5 a 7 vezes ao dia, seguido de uma menor ingestão semanal (9,0\%).

A ingestão dos alimentos pertencentes ao grupo das hortaliças e vegetais foi relatada com frequência de 1 a 2 vezes diariamente por 50,0\% dos jovens;
40,9\% consomem apenas semanalmente, entre 2 e 5 vezes, e 9,1\% dos alunos ingerem vegetais somente mensalmente.

Com relação ao grupo das frutas, 63,3\% dos estudantes relataram consumir entre 2 e 5 vezes durante a semana, e apenas 31,8\% deles ingerem alimentos deste grupo diariamente.

No grupo dos produtos lácteos, $81,8 \%$ dos estudantes afirmaram consumir pelo menos uma vez ao dia alimentos na forma de leite, queijo, iogurte e derivados.

Ao analisar o grupo das carnes, a maioria dos jovens $(72,7 \%)$ tem preferência pelo consumo de carne bovina, e a ingerem com frequência semanal; $63,6 \%$ consomem aves mensalmente, e 45,4\% relataram que o peixe faz parte da composição mensal de suas refeições.

Quanto ao grupo do feijão e leguminosas, $68,1 \%$ dos entrevistados relatam comer diariamente feijão e/ou leguminosas, variando de 1 a 2 vezes nas 24 horas.

A frequência de ingestão do grupo das gorduras, óleos e açúcares foi de 81,8\% entre os universitários, com frequência entre 1 e 3 vezes ao dia; somente $18,1 \%$ dos entrevistados ingerem alimentos deste grupo apenas semanalmente.

Ao serem questionados sobre o hábito de adicionar sal aos alimentos depois de preparados, 59,0\% dos jovens relataram não ter este hábito, enquanto $27,2 \%$ deles adicionam "às vezes", e 13,6\% afirmaram "sempre" adicionar sal ao alimento depois de pronto. 
Todos os entrevistados afirmaram consumir água diariamente, em média, sete vezes, mas não sabem referir a exata quantidade ingerida. A maioria $(63,6 \%)$ relatou não consumir bebidas alcoólicas. Os outros entrevistados indicam consumir álcool apenas semanalmente $(18,1 \%)$, ou mensalmente $(22,3 \%)$.

\section{Discussão}

A região amazônica apresenta uma diversidade ecológica, cultural e alimentar muito vasta, no entanto, este espaço ainda é pouco explorado em termos de pesquisas científicas, dificultando o adequado entendimento desta região e suas especificidades. Ao se tratar dos hábitos alimentares de sua população, particularmente dos jovens, torna-se mais evidente a escassez de estudos, não havendo investigações disponíveis sobre este tema em estudantes do ensino superior. Por isso, o conhecimento dos hábitos alimentares dos universitários desta região pode ser uma ferramenta importante para compreender o cenário epidemiológico amazônico e para a elaboração de estratégias e políticas públicas mais efetivas.

Nota-se que os hábitos alimentares dos universitários entrevistados são delineados por uma deficiência do consumo de alimentos pertencentes aos grupos das frutas, hortaliças e verduras, e excesso da ingestão de alimentos do grupo das gorduras, óleos e açúcares. Resultados semelhantes foram encontrados por Vieira e colaboradores (2002) ao analisar o perfil nutricional de adolescentes recém-ingressos em uma universidade pública brasileira. Naquele estudo, 79,5\% e $25,4 \%$ dos estudantes relataram que o grupo das hortaliças e frutas, respectivamente, são os mais rejeitados por eles além de referirem a ingestão frequente de alimentos doces e gordurosos.

De acordo com Alves e Boog (2007), em um estudo com 825 universitários sobre o comportamento alimentar em moradia estudantil no município de Campinas, São Paulo, a maioria dos estudantes universitários relacionou a má alimentação ao fato de não ter uma companhia na hora das refeições. Em pesquisa semelhante, estudantes afirmaram que realizar as principais refeições acompanhados da família, principalmente, favorece na escolha de alimentos mais saudáveis (Feitosa et al., 2010).

A maioria dos estudantes entrevistados em Belém relatou fazer suas refeições na universidade visto que é a solução mais fácil para quem necessita manter-se no ambiente de estudo. Em função do tempo dedicado às atividades escolares, abreviaram-se os rituais destinados à alimentação, deslocando-os para ambientes compatíveis com outras atividades, recorrendo à alimentação 
coletiva, em refeitórios ou restaurantes, principalmente localizados no espaço universitário (Garcia, 1997; Martins, 2009).

Foram encontradas diferenças consideráveis quanto ao número de refeições realizadas diariamente, destacando que a maioria faz de 3 a 4 refeições, sendo que as principais são desjejum, almoço e jantar. Gambardella e colaboradores (1999), em estudo sobre a prática alimentar de adolescentes da região de Santo André, São Paulo, destacam que o desjejum era a refeição mais negligenciada por $18,0 \%$ dos jovens, e 6,0\% dos entrevistados por eles substituíam o jantar por lanches ou comidas prontas. No Brasil, há estudos que demonstram que universitários substituem o almoço e o jantar por lanches, principalmente quando este já é o hábito familiar (Garcia et al., 2012). Os lanches e refeições rápidas geralmente são alimentos mais ricos em gorduras e carboidratos, e pobres em vitaminas, sais minerais e fibras.

Vieira e colaboradores (2002), em estudo do comportamento alimentar de recém-ingressos em uma universidade pública brasileira (que não foi nomeada), verificaram que $60,0 \%$ dos entrevistados não tinham o hábito de realizar as três refeições ao dia (desjejum, almoço e jantar). Naquele estudo apenas 15,4\% dos alunos realizavam o jantar com os mesmos alimentos presentes no almoço, havendo comumente substituição por lanches, e 37,0\% não realizavam o desjejum.

Segundo o Guia Alimentar para a População Brasileira, para garantir a saúde, é necessário fazer pelo menos três refeições por dia (café da manhã, almoço e jantar), intercaladas por pequenos lanches (Brasil, 2008).

Quanto aos horários entre refeições, houve prevalência $(59,0 \%)$ do intervalo de 3 a 4 horas entre uma refeição e outra. Porém, 4,5\% dos jovens relataram ficar até 9 horas sem se alimentar. Resultados semelhantes foram encontrados em pesquisa realizada em duas instituições diferentes em uma universidade de Portugal, em que a maioria $(87,6 \%)$ dos jovens entrevistados mantinha um intervalo de 3 horas entre as refeições (Garcia et al., 2012). O jejum prolongado e o baixo número de refeições podem promover hipotrofia do estômago e intestino delgado, com isso os alimentos são mal digeridos e mal absorvidos, acarretando em complicações à saúde como doenças gástricas ou até mesmo obesidade (Brasil, 2008; Hu, 2008; Varela et al., 2007).

Embora boa parcela dos entrevistados neste estudo $(40,9 \%)$ tenha demonstrado interesse no valor nutricional dos alimentos no momento da compra, a maioria (59,1\%) relatou não se preocupar com tal questão. No estudo realizado por Machado e colaboradores (2006), em supermercados e hipermercados de Feira de Santana, Bahia, com 300 consumidores de ambos os sexos, com faixa etária predominante de 25 a 30 anos, consta- 
tou-se que apenas 2,0\% dos entrevistados se preocupavam com a composição nutricional do produto na hora de adquiri-lo, o que indica que os principais determinantes das escolhas alimentares de uma população, inclusive de universitários (Martins, 2009), dependem de uma ampla gama de fatores.

Em Belém, a ingestão dos alimentos do grupo dos cereais mostra-se dentro do que é recomendado pelo Guia Alimentar para a População Brasileira (Brasil, 2008), de cinco a nove porções diárias, mostrando que os carboidratos comumente fazem parte da alimentação desses jovens. Este grupo de nutrientes fornece energia às células do corpo, particularmente ao cérebro, visto o elevado número de atividades que requerem esforço físico e mental dos estudantes durante o seu dia (Maihara et al., 2006).

A ingestão de hortaliças e vegetais mostra-se deficiente quando comparada com as recomendações de, no mínimo, cinco porções diárias do Guia Alimentar para a População Brasileira (Brasil, 2008). Uma das principais razões para a ingestão diária, ainda que limitada, dos alimentos desse grupo é que eles fazem parte do cardápio tradicional do restaurante universitário, que alguns dos entrevistados utilizam diariamente. O consumo diário e variado de tais alimentos é importante na composição de uma dieta balanceada, pois são fontes de micronutrientes, fibras e de outros com- ponentes com propriedades funcionais, e possuem baixa densidade energética, favorecendo a manutenção saudável do peso corporal (Figueiredo et al., 2008).

Observou-se, também, uma ingestão inadequada de frutas por parte dos jovens entrevistados, que chegam a consumi-las apenas mensalmente ou semanalmente.

Os hábitos alimentares dos jovens têm passado por diversas modificações, observando mudanças no padrão alimentar, como o baixo consumo de frutas, hortaliças e verduras, e a substituição das principais refeições por alimentos industrializados com elevados teores calóricos, podendo ocasionar o aparecimento de comorbidades associadas, comprometendo a saúde em longo prazo (Falcão et al., 2007; Nobre et al., 2012). Esta é uma questão que merece atenção, pois o recomendado, segundo Philippi (2008), é pelo menos 3 porções por dia de frutas, pois estas auxiliam na prevenção de determinadas deficiências nutricionais e evitam o desenvolvimento de doenças crônicas.

Embora haja o consumo diário de leite e derivados pela maioria dos universitários entrevistados, o mesmo não se encontra dentro do que é preconizado pelas recomendações diárias, que seria de 3 porções. O leite e seus derivados são importantes fontes de micronutrientes, como riboflavina (vitamina $\mathrm{B}_{2}$ ), vitamina D e principal fonte de cálcio na alimen- 
tação, e sua deficiência pode resultar em alguns prejuízos à saúde, como retardo de crescimento, fraqueza muscular, deformidade esquelética e osteoporose (Bueno e Czepielewski, 2008).

No grupo das carnes, seu consumo em Belém encontrou-se dentro do esperado de acordo com as recomendações diárias (1 a 2 porções), contribuindo para a manutenção da saúde visto que os alimentos deste grupo, de modo geral, são boas fontes de todos os aminoácidos essenciais, substâncias químicas que compõem as proteínas, necessárias para o crescimento e a manutenção do corpo humano, bem como são fontes importantes de ferro de alta biodisponibilidade e vitamina $B_{12}$ (Brasil, 2008).

A adequada ingestão de feijão ou outros grãos diariamente pela maioria dos estudantes é importante, pois são fontes naturais de fibras alimentares e contêm aminoácidos essenciais, vitaminas e minerais, que devem compor a alimentação básica de todos os brasileiros. Entre os alimentos de consumo diário, o feijão é a maior fonte de fibra da dieta habitual e, juntamente com o arroz, é considerado item básico do padrão alimentar brasileiro (Marinho et al., 2007; Mattos e Martins, 2000). Em pesquisa realizada por Rodrigues e colaboradores (2012) com estudantes do ensino médio público e privado, na faixa etária de 14 a 19 anos, no município de Cuiabá, foi demonstrado que o padrão "tradicional", composto

principalmente por alimentos típicos da dieta tradicional brasileira, como o arroz e o feijão, apresentou maior aderência entre os adolescentes sem excesso de peso, ressaltando que, em outros estudos, esta combinação tem se mostrado o principal fator protetor contra a obesidade (Brasil, 2008; Nascimento et al., 2011).

A alta prevalência do consumo de gorduras, óleos e açúcares diariamente reflete hábitos errôneos na população investigada. O elevado consumo de dietas ricas em gorduras e açúcares, quando associado ao aumento do sedentarismo, em parte devido às longas horas de estudo, aumenta o risco de doenças crônicas não-transmissíveis e a prevalência de obesidade, o que pode gerar importantes impactos futuros no perfil de morbimortalidade do estado e do país (Neutzling et al., 2007).

O hábito de adicionar sal após o alimento estar pronto para o consumo faz parte da rotina dos jovens amazônidas, podendo ultrapassar o limite recomendado de $5 \mathrm{~g}$ de sal por dia (Brasil, 2008). Embora presente naturalmente em diversos alimentos em quantidades que atendem às necessidades humanas, a maior parte do sódio da dieta é proveniente dos compostos sódicos adicionados no processamento dos alimentos ou, em menor escala, do sal de mesa, sendo preditor de agravos à saúde, particularmente associado aos níveis pressóricos (Molina et al., 2003; Rique et al., 2002). 
A maioria dos estudantes indicou ter consumo adequado de água, tratando-se de um resultado importante, pois a água é necessidade primordial para a vida e recurso natural indispensável ao ser humano (Scuracchio e Farache Filho, 2011), sendo essencial para o bom funcionamento do organismo. A maioria das atividades metabólicas depende de água para funcionar adequadamente, assim como ela é usada no corpo para o transporte de compostos hidrossolúveis como macro e micronutrientes, metabólitos e excretas (Figueiredo et al., 2008).

\section{Conclusões}

Conhecer o comportamento alimentar dos diversos segmentos demográficos e estratos sociais da região amazônica, como os ribeirinhos, quilombolas, indígenas, e também os universitários e outros grupos urbanos, é fundamental para o planejamento de políticas públicas específicas visando conter a escalada das doenças crônicas no Brasil.

No caso específico dos universitários, parcela potencialmente mais letrada do país, faz-se necessário maior atenção à educação em relação aos hábitos alimentares saudáveis, mesmo considerando que são pessoas com um cotidiano atribulado, que comumente costumam se alimentar fora de casa e têm tempo bastante reduzido para as refeições, implicando em uma alimentação menos saudável em relação àquelas pessoas que comem no âmbito familiar/ doméstico. $O$ trabalho integrado de nutricionistas e bioantropólogos na área de saúde coletiva pode ser fundamental para ajudar a compreender e modificar para melhor os padrões alimentares dos jovens estudantes.

\section{Referências bibliográficas}

Alves, H. J.; Boog, M. C. F. 2007. Comportamento Alimentar em Moradia Estudantil: Um Espaço para Promoção da Saúde. Revista de Saúde Pública, 41(2): 197-204. DOl: 10.1590/S0034-89102007000200005.

Azevedo, R. C. S.; Barreto, R. A.; Campos, L. M. R. M. C.; Gasparini, M. R.; Nascimento-Filho, G. A.; Melo, L. S.; Lima, R. M. 2008. Hábitos Alimentares na Comunidade Universitária do ISECENSA. Revista Perspectivas [Online], 5(1): 126-137. [Consultado em 20-5-2013]. Disponível em: http://www.seer.perspectivasonline.com.br/index.php/revista_antiga/article/view/293.

Bardin, L. 1977. Análise de Conteúdo. Lisboa, Edições 70.

Brasil. 2008. Guia Alimentar para a População Brasileira: Promovendo a Alimentação Saudável. Brasília, Ministério da Saúde, Secretaria de Atenção à Saúde. 
Bueno, A. L; Czepielewski, M. A. 2008. The Importance for Growth of Dietary Intake of Calcium and Vitamin D. Jornal de Pediatria, 84(5): 386-394. DOI: 10.2223/ JPED.1816.

Falcão, V. T. F. L.; Miranda, M. L.; Silva, R. M. C. 2007. Prevalência de Obesidade e Sobrepeso entre os Universitários do Campus de Saúde da Universidade de Pernambuco. Revista da Rede de Enfermagem do Nordeste, Fortaleza, 8(3): 1725. Disponível em http://www.revistarene.ufc.br/revista/index.php/revista/ article/view/663/pdf.

Feitosa, E. P. S.; Dantas, C. A. O.; Andrade-Wartha, E. R. S.; Marcellini, P. S.; Mendes-Netto, R. S. 2010. Hábitos Alimentares de Estudantes de uma Universidade Pública no Nordeste, Brasil. Alimentos e Nutrição, 21(2): 225-230. Disponível em: http:// serv-bib.fcfar.unesp.br/seer/index.php/ alimentos/article/viewArticle/1185.

Figueiredo, I. C. R.; Jaime, P. C.; Monteiro, C. A. 2008. Fatores Associados ao Consumo de Frutas, Legumes e Verduras em Adultos da Cidade de São Paulo. Revista de Saúde Pública, 42(5): 777-85. DOI: 10.1590/S0034-89102008005000049.

Gambardella, A. M. D.; Frutuoso, M. F. P.; Franchi, C. 1999. Prática Alimentar de Adolescen- tes. Revista de Nutrição, 12(1): 5-19. DOl: 10.1590/S1415-52731999000100005.

Garcia, R. W. D. 1997. Práticas e Comportamento Alimentar no Meio Urbano: Um Estudo no Centro da Cidade de São Paulo. Cadernos de Saúde Pública, 13(3): 455-467. DOI: 10.1590/S0102-311X1997000300021.

Garcia, D.; Nabo, G.; Correia, V.; Ribeiro, l.; Costa, M. C. 2012. Atividade Física e Hábitos Alimentares numa População Universitária. Biomedical and Biopharmaceutical Research, 2(9): 147-158.

Guerra, I. C. 2008. Pesquisa Qualitativa e Análise de Conteúdo: Sentidos e Formas de Uso. Lisboa, Princípia.

Hu, F. B. 2008. Obesity Epidemiology. Cambridge, Oxford University Press.

Krippendorff, K. 2003. Content Analysis, An Introduction to Its Methodology, $2^{\text {nd }}$ ed. London, Sage Publications.

Machado, S. S.; Santos, F. O.; Albinati, F. L.; Santos, L. P. R. 2006. Comportamento dos Consumidores com Relação à Leitura de Rótulo de Produtos Alimentícios. Alimentos e Nutrição, 17(1): 97-103. Disponível em: http://serv-bib.fcfar. unesp.br/seer/index.php/alimentos/ article/viewArticle/119.

Maihara, V. A.; Silva, M. G.; Baldini, V. L. S.; Miguel, A. M. L.; Fávaro, D. I. T. 2006. Avaliação Nutricional de Dietas de Trabalhadores em Relação a Proteínas, Lipídeos, Carboidratos, Fibras alimentares e Vitaminas. Ciência e Tecnologia de Alimentos, 26(3): 672-677. DOl: 10.1590/S0101-20612006000300029. 
Marinho, M. C. S.; Hamann, E. M.; Lima, A. C. F. 2007. Práticas e Mudanças no Comportamento Alimentar na População de Brasília, Distrito Federal, Brasil. Revista Brasileira de Saúde Materno Infantil, 7(3): 251-261. DOI: 10.1590/S151938292007000300004.

Martins, M. J. R. L. 2009. Hábitos Alimentares de Estudantes Universitários. Tese de Licenciatura, Universidade do Porto, Portugal, Faculdade de Ciências da Nutrição e Alimentação. [Consultado em 24-10-2011].

Mason, M. 2010. Sample Size and Saturation in PhD Studies Using Qualitative Interviews. Forum: Qualitative Social Research, 11(3). [Consultado em 193-2014]. Disponível em: http://www. qualitative-research.net/index.php/ fqs/article/view/1428/3027

Mattos, L. L.; Martins, I. S. 2000. Consumo de Fibras Alimentares em População Adulta. Revistade SaúdePública, 34(1):50-55. DOI: 10.1590/S1519-38292007000300004.

Molina, M. C. B.; Cunha, R. S.; Herkenhoff, L. F.; Mill, J. G. 2003. Hipertensão Arterial e Consumo de Sal em População Urbana. Revista de Saúde Pública, 37(6): 743-50. DOI: 10.1590/S00348910200300060000.

Nascimento, S.; Barbosa, F. S.; Sichieri, R.; Pereira, R. A. 2011. Dietary Availability Patterns of the Brazilian Macro-regions. Nutrition Journal, 10: 79. DOl: 10.1186/1475-2891-10-79.

Neutzling, M. B; Araújo, C. L. P.; Vieira, M. F. A.; Hallal, P. C.; Menezes, A. M. B. 2007. Fre- qüência de Consumo de Dietas Ricas em Gordura e Pobres em Fibra entre Adolescentes. Revista de Saúde Pública, 41(3): 336-342. DOI: 10.1590/S003489102007000300003.

Neutzling, M. B.; Assunção, M. C. F.; Malcon, M. C.; Hallal, P. C.; Menezes, A. M. B. 2010. Hábitos Alimentares de Escolares Adolescentes de Pelotas, Brasil. Revista de Nutrição, 23(3): 379-388. DOI: 10.1590/ S1415-52732010000300006.

Nobre, L. N.; Lamounier, J. A; Franceschini, S. C. C. 2012. Preschool Children Dietary Patterns and Associated Factors. Jornal de Pediatria, 88(2): 129- 136. DOI: 10.2223/JPED.2169.

Oliveira, A. S.; Silva, V. A. P.; Alves, J. J.; Fagundes, D.; Pires, I. S. C.; Miranda, L. S. 2012. Mães e Amamentação Versus Hábitos Alimentares de Pré-escolares. Alimentos e Nutrição, 23(3): 377-386. Disponível em: http://serv-bib.fcfar.unesp. br/seer/index.php/alimentos/article/ viewFile/377/1693.

Philippi, S. T. 2008. Pirâmide dos Alimentos: Fundamentos Básicos da Nutrição. Barueri, São Paulo, Manole.

Rique, A. B. R.; Soares, E. A.; Meirelles, C. M. 2002. Nutrição e Exercício na Prevenção e Controle das Doenças Cardiovasculares. Revista Brasileira de Medicina e Esporte, 8 (6): 224-254. DOI: 10.1590/S1517-86922002000600006.

Rodrigues, P. R. M.; Pereira, R. A.; Cunha, D. B.; Sichieri, R.; Ferreira, M. G.; Vilela, A. A. F.; Gonçalves-Silva, R. M. V. 2012. Fa- 
tores Associados a Padrões Alimentares em Adolescentes: Um Estudo de Base Escolar em Cuiabá, Mato Grosso. Revista Brasileira de Epidemiologia, 15(3): 662-674. DOI: 10.1590/S1415$-790 \times 2012000300019$

Scuracchio, P. A.; Farache Filho, A. 2011. Qualidade da Água Utilizada para Consumo em Escolas e Creches no Município de São Carlos - SP. Alimentos e Nutrição, 22(4): 641-647. Disponível em: http://serv-bib. fcfar.unesp.br/seer/index.php/alimentos/article/viewArticle/1495.

Varela, A. L.; Quintans, C. C.; Tranqueira, A. P. M.; Gasparotto, R.; Isaac, I. A. S.; Estrela, R. A. M.; Costa, F. M. C. B.; Campos, A. A. M. S. 2007. Programa de Emagrecimento para Mulheres Obesas Envolvendo Variáveis Nutricionais, Psicológicas e Exercício Físico. Revista Brasileira de Obesidade, Nutrição e Emagrecimento, 1(6): 12-27. Disponível em: http://www.rbone.com.br/index.php/rbone/article/ view/54.

Vieira, V. C. R.; Priore, S. E.; Ribeiro, S. M. R.; Franceschini, S. C. C.; Almeida, L. P. 2002. Perfil Socioeconômico, Nutricional e de Saúde de Adolescentes Recém-ingressos em uma Universidade Pública Brasileira. Revista de Nutrição, 15(3): 273-282. DOl: 10.1590/S1415-52732002000300003. 